OPEN ACCESS

Edited by:

Miguel Cacho Teixeira,

Universidade de Lisboa, Portugal

Reviewed by:

Elena Baraldi,

Università degli Studi di Bologna, Italy

Jon Y. Takemoto,

Utah State University, United States

${ }^{*}$ Correspondence:

Rohan G. T. Lowe

r.lowe@latrobe.edu.au

Specialty section:

This article was submitted to Antimicrobials, Resistance

and Chemotherapy,

a section of the journal

Frontiers in Microbiology

Received: 03 May 2018

Accepted: 02 July 2018

Published: 24 July 2018

Citation

McColl Al, Bleackley MR,

Anderson MA and Lowe RGT (2018)

Resistance to the Plant Defensin

NaD1 Features Modifications to the Cell Wall and Osmo-Regulation

Pathways of Yeast.

Front. Microbiol. 9:1648

doi: 10.3389/fmicb.2018.01648

\section{Resistance to the Plant Defensin NaD1 Features Modifications to the Cell Wall and Osmo-Regulation Pathways of Yeast}

\author{
Amanda I. McColl, Mark R. Bleackley, Marilyn A. Anderson and Rohan G. T. Lowe* \\ Department of Biochemistry and Genetics, La Trobe Institute for Molecular Science, La Trobe University, Melbourne, VIC, \\ Australia
}

Over the last few decades, the emergence of resistance to commonly used antifungal molecules has become a major barrier to effective treatment of recurrent life-threatening fungal diseases. Resistance combined with the increased incidence of fungal diseases has created the need for new antifungals, such as the plant defensin NaD1, with different mechanisms of action to broaden treatment options. Antimicrobial peptides produced in plants and animals are promising new molecules in the arsenal of antifungal agents because they have different mechanisms of action to current antifungals and are often targeted specifically to fungal pathogens (van der Weerden et al., 2013). A key step in the development of novel antifungals is an understanding of the potential for the fungus to develop resistance. Here, we have used the prototypic plant defensin NaD1 in serial passages with the model fungus Saccharomyces cerevisiae to examine the evolution of resistance to plant antifungal peptides. The yeast strains did develop tolerance to NaD1, but it occurred more slowly than to the clinically used antifungal caspofungin. Sequencing the genomes of the strains with increased tolerance failed to identify any 'hotspot' mutations associated with increased tolerance to NaD1 and led to the identification of 12 genes that are involved in resistance. Characterization of the strains with increased tolerance to NaD1 also revealed changes in tolerance to abiotic stressors. Resistance developed slowly via an accumulation of single nucleotide mutations and had a fitness penalty associated with it. One of the genes identified FPS1, revealed that there is a common mechanism of resistance to NaD1 that involves the osmotic stress response pathway. These data indicate that it is more difficult to generate resistance to antimicrobial peptides such as NaD1 compared to small molecule antifungals.

Keywords: antifungal, defensin, genome, yeast, resistance, NaD1, cell wall, stress

\section{INTRODUCTION}

Pathogenic fungi have become a serious threat to both agriculture and human health (Murray and Brennan, 2009). In human health, fungal pathogens are detrimental to immunocompromised individuals, such as individuals with HIV, transplant recipients and cancer patients receiving chemotherapy (Ortega et al., 2010). Indeed, some invasive fungal diseases can become 
life-threatening in the immunocompromised and mortality can reach up to $80 \%$ (Lass-Flörl, 2009). There are very few therapeutic options for systemic fungal infections, and some fungicides are known to be dangerous to human health due to severe side effects such as toxicity (Ortega et al., 2010). Fungicide resistance occurs when a fungal pathogen becomes less susceptible to an antifungal agent. Resistance is broadly characterized by the mechanism by which it occurs. These mechanisms include; alteration of the target site in a protein, detoxification of the fungicide, overexpression of the target site, and the use of efflux pumps to expel the fungicide (Leroux et al., 2002). The increased use of the small molecule antifungal drugs that are currently in the clinic as well as related molecules used in agriculture has led to reports of fungal pathogens resistant to almost all common antifungals (Verweij et al., 2009). There is a need for new antifungal agents to battle the phenomenon of fungal resistance; antifungal proteins are one attractive option for development (Sanglard et al., 1996; van der Weerden et al., 2013).

A wide variety of organisms produce antifungal peptides as part of their innate immunity arsenal (van der Weerden et al., 2013). They are highly represented in plants where defensins are the largest family. Plant defensins are small proteins of 45 to 54 amino acids that are ubiquitous in the seeds, leaves and flowers of all plants examined (Berkut et al., 2014). They are usually produced constitutively as a defense against pathogens, particularly in reproductive tissues and seeds (Lay et al., 2003). They are also expressed in response to infections and environmental stress (Lay et al., 2003; Sagaram et al., 2011). There are thousands of plant defensins in public sequence databases. They share a common structure, but are highly variable in sequence and, not surprisingly, they often have different mechanisms of action (Parisi et al., 2018). The mechanism of action of only a handful of defensins has been elucidated. They often have multistep mechanisms that affect more than one target in the fungus (Parisi et al., 2018). Hence, it is expected that resistance to defensins is likely to develop more slowly than resistance to smaller antifungal molecules that interact with a single site, composed of a few amino acids, on a single protein target. $\mathrm{NaD} 1$ is a potent antifungal defensin that accumulates in the flowers of the ornamental tobacco plant Nicotiana alata, where it functions to protect the reproductive organs from damage by fungal pathogens (Lay et al., 2003). NaD1 has a wellcharacterized structure, and several features of its mechanism of action have been well described but not completely elucidated (Lay et al., 2012). NaD1 has at least a three-step mechanism of action that involves: interaction with the fungal cell wall (van der Weerden et al., 2008), movement across the plasma membrane, induction of oxidative stress, and interaction with phosphatidylinositol 4,5 bisphosphate. These processes lead to damage of the inner leaflet of the cell membrane and cell death within 10 min of exposure to NaD1 (van der Weerden et al., 2010; Hayes et al., 2014; Poon et al., 2014; Payne et al., 2016).

In this study, yeast strains were generated that have increased tolerance to $\mathrm{NaD} 1$, and genetic mutations linked to the decreased response to $\mathrm{NaD} 1$ were identified. Phenotypic characterization of resistant lines revealed slower growth rates, as well as cell wall changes reflected as sensitivity to the anionic detergent SDS and the chitin binding molecule calcofluor white (CFW). That is, there was a fitness trade-off associated with NaD1-resistance. Mutations across twelve genes correlated with NaD1 resistance. These genes were associated with diverse aspects of cellular processes suggesting that $\mathrm{NaD} 1$ acts upon multiple cellular targets. Affected locations or processes included the cell wall, transporters and signaling pathways. Mutations in the gene FPS1 indicate glycerol accumulation may modulate $\mathrm{NaD} 1$ antifungal activity. Resistance to NaD1 occurred more slowly than resistance to caspofungin in similar experiments.

\section{MATERIALS AND METHODS}

\section{Fungal Strains}

The S. cerevisiae strain BY4741 (MATahis3 $\Delta 0$ leu2 $\Delta 0$ met $15 \Delta 0$ ura $3 \Delta 0$ ) was purchased from Thermo Scientific. Single deletion strains were retrieved from the haploid non-essential deletion collection (Thermo Scientific) (Winzeler et al., 1999). S. cerevisiae was routinely cultured on YPD-Agar (1\% yeast extract, 2\% peptone, $2 \%$ dextrose, $2 \%$ agar) medium at $30^{\circ} \mathrm{C}$.

\section{Antifungal Molecules}

$\mathrm{NaD} 1$ and $\mathrm{NaD} 2$ were purified from Nicotiana alata flowers as described in Lay et al. (2003) and Dracatos et al. (2014). HXP4 and DmAMP1 were expressed in Pichia pastoris and purified as described previously (Hayes et al., 2013; Bleackley et al., 2016). CP29 was purchased from GL Biochem (China), BPTI (synonym Aprotinin) was purchased from Astral Scientific (Australia), caspofungin was purchased from Sigma (Australia).

\section{Culturing in the Presence of Antifungal Molecules to Develop Resistance}

S. cerevisiae $\mathrm{BY} 4741$ was grown overnight at $30^{\circ} \mathrm{C}$ with agitation in $5 \mathrm{~mL}$ of YPD. The overnight culture was then diluted to an OD $600 \mathrm{~nm}$ of 0.01 in $50 \%$ strength PDB medium $(1 / 2$ PDB) before addition of antifungal molecules. Cultures were initially grown with the antifungal molecules at $0.5 \mathrm{x}$ the minimum inhibitory concentration (MIC) or 1x MIC alongside a negative control lacking antifungals. Three independent lines for the test and controls were grown at the same time. The cultures were incubated overnight at $30^{\circ} \mathrm{C}$ with agitation. The cultures that exhibited growth at the highest concentration of the antifungal molecules were sub-cultured with medium containing a higher concentration of the antifungal molecule. Sub-culturing was stopped once growth occurred at 32 times the original MIC.

\section{Single-Colony Isolation of Resistant Strains}

Cultures that were more tolerant to the antifungal molecule were streaked out for single colonies on non-selective YPD agar. Three colonies were picked from each line, and their resistance was re-tested. The colony with the highest resistance to the antifungal was retained for further experimentation. The MIC of pure strains isolated from each culture was broadly equivalent (Supplementary Figure 1). 


\section{Antifungal Assay}

Antifungal assays were performed as described in Hayes et al. (2013). Briefly, cultures were grown overnight $\left(30^{\circ} \mathrm{C}, 250 \mathrm{rpm}\right)$ in $5 \mathrm{~mL}$ YPD and diluted to an OD600 of $0.01 \mathrm{in}^{1} / 2$ PDB. Antifungal molecules were prepared at $10 \mathrm{x}$ the assay concentration, and $10 \mu \mathrm{L}$ was mixed with $90 \mu \mathrm{L}$ of diluted yeast culture before incubation for $24 \mathrm{~h}$ at $30^{\circ} \mathrm{C}$. The final OD600 was measured using a SpectraMAX M5e plate reader (Molecular Devices).

\section{Cell Growth Assay}

S. cerevisiae BY4741 cultures were grown overnight $\left(30^{\circ} \mathrm{C}, 250\right.$ $\mathrm{rpm})$ in $5 \mathrm{~mL}$ of YPD and diluted to an $\mathrm{OD}_{600}$ of $0.5 \mathrm{in} 1 \mathrm{~mL}$ YPD and $1 / 2$ PDB. Each culture $(100 \mu \mathrm{L})$ was incubated in a SpectraMAX M5e plate reader (Molecular Devices) at $30^{\circ} \mathrm{C}$ in a 96-well microtiter plate format. Optical density at $600 \mathrm{~nm}$ was recorded every $30 \mathrm{~min}$ over the $48 \mathrm{~h}$ culture period.

\section{Cell Size and Area Measurement}

S. cerevisiae BY4741 cultures were grown overnight $\left(30^{\circ} \mathrm{C}, 250\right.$ $\mathrm{rpm})$ in $5 \mathrm{~mL}$ of YPD and were imaged using an Olympus IX81 brightfield microscope (LIMS Bioimaging Facility). Cell dimensions were measured from images using FIJI software (Schindelin et al., 2012). A minimum of 30 cells was measured for each sample.

\section{Stress Assay With Hydrogen Peroxide, Calcofluor White, $\mathrm{NaCl}$, and SDS}

YPD agar medium $(25 \mathrm{~mL})$ was amended to a final concentration of hydrogen peroxide (0.625 mM, $1.25 \mathrm{mM}, 2.5 \mathrm{mM}, 5 \mathrm{mM})$, CFW $(1 \mu \mathrm{g} / \mathrm{mL}, 2.5 \mu \mathrm{g} / \mathrm{mL}, 5 \mu \mathrm{g} / \mathrm{mL}, 10 \mu \mathrm{g} / \mathrm{mL}), \mathrm{NaCl}(100$ $\mathrm{mM}, 200 \mathrm{mM}, 300 \mathrm{mM})$, or SDS $(12.5 \mu \mathrm{g} / \mathrm{mL}, 25 \mu \mathrm{g} / \mathrm{mL}, 50$ $\mu \mathrm{g} / \mathrm{mL}, 100 \mu \mathrm{g} / \mathrm{mL})$ just before each plate was poured. Yeast cultures were grown overnight in $5 \mathrm{~mL}$ of YPD before dilution to an OD $600 \mathrm{~nm}$ of 0.1. A fivefold dilution series of each culture was spotted onto the plate ( $4 \mu \mathrm{L}$ per spot) and incubated overnight at $30^{\circ} \mathrm{C}$ before being photographed.

\section{Stress Assay With Ultraviolet Light}

$S$. cerevisiae cultures were grown overnight in $5 \mathrm{~mL}$ YPD and diluted to an OD $600 \mathrm{~nm}$ of 0.1 in $1 \mathrm{~mL}$ MilliQ-purified water. A fivefold dilution series of each strain $(4 \mu \mathrm{L}$ per spot) was added to the YPD agar plate and allowed to dry, before exposure to UV light (Phillips, $30 \mathrm{~W}$ bulb at $50 \mathrm{~cm}$ ) for 1.2, 2.4, 5.2, or $10.4 \mathrm{~min}$.

\section{Stress Assay With Heat}

S. cerevisiae cells were grown overnight in $5 \mathrm{~mL}$ YPD and diluted to an OD $600 \mathrm{~nm}$ of $0.1 \mathrm{in} 1 \mathrm{~mL}$ MilliQ-purified water. Diluted cultures $(100 \mu \mathrm{L})$ were heated $\left(30^{\circ} \mathrm{C}, 37^{\circ} \mathrm{C}, 41^{\circ} \mathrm{C}\right.$, or $\left.46^{\circ} \mathrm{C}\right)$ for 30 min. Survival was assessed after heat treatment using a spot assay on YPD agar.

\section{DNA Extraction From Wild-Type and Resistant Strains of S. cerevisiae}

Genomic DNA was extracted using the Qiagen DNeasy ${ }^{\circledR}$ plant miniprep kit. Three individual lines of NaD1-resistant strains and three lines of the no-treatment controls were sequenced.
Sequencing was completed at the La Trobe Genomics Platform, using Illumina MiSeq V3 chemistry. One run was performed for all six genomes, generating 25 million 300 bp paired-end reads. The pre-processing and variant discovery steps were performed as described by the GATK best practices and are summarized in McKenna et al. (2010).

\section{Genomic Analysis of Resistant Strains of S. cerevisiae Sequence Pre-processing}

Picard tools (v.2.4.1) fastqtosam was used to convert raw sequence files into Sam format and to add read group information. Any Illumina adapters were identified and marked using Picard (v.2.4.1) markilluminaadapters. BWA-mem (v.0.7.12) was used to align reads to the reference S. cerevisiae (R64-1-1.23) genome (Engel et al., 2014). Alignment files were merged, and duplicate reads were marked using Picard (v.2.4.1) mergebamalignment and markduplicates. Local alignments were optimized, and sequence quality scores were recalibrated using GATK (v.3.6) realignertargetcreater and baserecalibrator.

\section{Variant Discovery}

GATK (v.3.6) Haplotypecaller was used to find genome variations that were either SNVs (single-nucleotide variants) or INDELs (insertion/deletion) simultaneously, also using known variants from dbSNP (Sherry et al., 2001). The samples were merged using GATK (v.3.6) combinegvcf, and then GenotypeGVCFs was used to rescore and genotype the combined gVCFs. GATK (v.3.6) VariantFiltration and VariantRecalibrator were used to extract SNVs and indels from the combined call set based on the default quality parameters, the SNVs and indels were then labeled as passed or filtered.

\section{Variant Refinement}

The high-quality variants identified during the variant discovery process were annotated using SnpEff (v.2.4) (Cingolani et al., 2012). SnpEff was used to determine whether each mutation was predicted to alter an encoded protein sequence (Table 3 ). Variant effect predictor (VEP) marked any codon changes as either tolerant or deleterious (McLaren et al., 2016). SnpSift (v.2.4) was used to was used to identify SNVs or indels that were present in NaD1 resistant replicates and not in the Control strains (Table 3). The variants selected during refinement were inspected manually using IGV (v.2.3.77) to rule out unexpected processing artifacts (Robinson et al., 2011).

\section{Sanger Sequencing of the FKS1 Gene of Caspofungin-Resistant Mutants}

The FKS1 gene from three individual lines of caspofunginresistant strains and a no treatment control was amplified by PCR using primers TCAAGGAAGGCAAGAAAAGCTA and GAGGCCGATACTGGTGAAAA and NEB Q5 proofreading polymerase according to the manufacturer's directions. Initial denaturation was at $95^{\circ} \mathrm{C}$ for $2 \mathrm{~min}$, followed by 30 cycles of: $95^{\circ} \mathrm{C} \quad 30 \mathrm{~s}, \quad 55^{\circ} \mathrm{C} \quad 30 \mathrm{~s}, \quad 72^{\circ} \mathrm{C} \quad 2 \mathrm{~min}$, and a final extension at $72^{\circ} \mathrm{C} 2 \mathrm{~min}$. Sanger sequencing of the FKS1 amplicon using primers "TCAAGGAAGGCAAGAAAAGCTA" 
and "CTGCATTTGCCCCTCTACAT" was completed by the Australian Genome Research Facility (AGRF). Sequence data were analyzed using Geneious software.

\section{RESULTS}

\section{Evolution of Resistance to NaD1}

Yeast strains with increased tolerance to $\mathrm{NaD} 1$ or caspofungin were developed by continuous culture of $S$. cerevisiae in sublethal concentrations of each antifungal molecule. Each time the MIC increased, the dose of antifungal was doubled. The starting concentration of $\mathrm{NaD} 1$ was $1 \mu \mathrm{M}$; it took 20 rounds of subculturing for NaD1-R A, 21 rounds for NaD1-R C and 22 rounds for NaD1-R B to achieve growth in $32 \mu \mathrm{M} \mathrm{NaD} 1$ (Figure 1A). In contrast, it took only 15 rounds of sub-culture to achieve growth in caspofungin at concentrations 32 -fold higher than the initial MIC $10 \mathrm{nM}$ (Figure 1A).

Three genetically pure strains of each of the NaD1-resistant and caspofungin-resistant lines were isolated, and their resistance phenotype was confirmed using a standard antifungal growth assay. The colony with the most resistance for each line was used for all further experimentation. The NaD1-resistant isolates were 10 -fold more resistant to $\mathrm{NaD} 1$ than the no-treatment control lines that had been passaged at the same time, with an MIC of $40 \mu \mathrm{M}$ compared to an MIC of $4 \mu \mathrm{M}$ (Figure 1B and Table 1). The caspofungin-resistant isolates were 25 -fold more resistant to caspofungin with an MIC of $500 \mathrm{nM}$ compared to the no treatment control which had an MIC of $20 \mathrm{nM}$ (Figure 1C and Table 1). In most fungal species, resistance to caspofungin occurs via mutations to the FKS1 gene within a "hot spot" zone affecting residues Phe639 to Pro647 (Katiyar and Edlind, 2009). Sequencing of the entire FKS1 gene of our caspofungin-resistant strains revealed that all three strains contained a single point mutation (F639V) confirming resistance was derived by the most commonly observed mechanism (Supplementary Figure 2).

\section{Resistance to NaD1 Confers Resistance to Some but Not All Antifungal Peptides}

The NaD1-resistant lines were tested against a range of antimicrobial molecules to determine if the observed resistance

TABLE 1 | The MIC of NaD1- and caspofungin-resistant lines of S. cerevisiae.

\begin{tabular}{lcc}
\hline Strain & NaD1 MIC $(\boldsymbol{\mu} \mathbf{M})$ & Caspofungin MIC (nM) \\
\hline Wild-type & 4 & 20 \\
NaD1-R A & 40 & 25 \\
NaD1-R B & 40 & 25 \\
NaD1-R C & 40 & 25 \\
Caspofungin-R A & 4 & 500 \\
Caspofungin-R B & 4 & 500 \\
Caspofungin-R C & 4 & 500 \\
\hline
\end{tabular}

The minimum inhibitory concentration (MIC) of $\mathrm{NaD1}$ and caspofungin are summarized for NaD1 resistant isolates, caspofungin resistant isolates and the parental wild type line of $S$. cerevisiae.

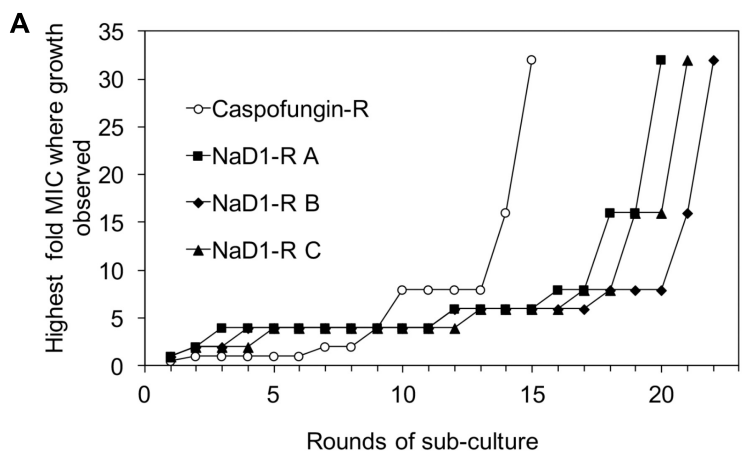

B
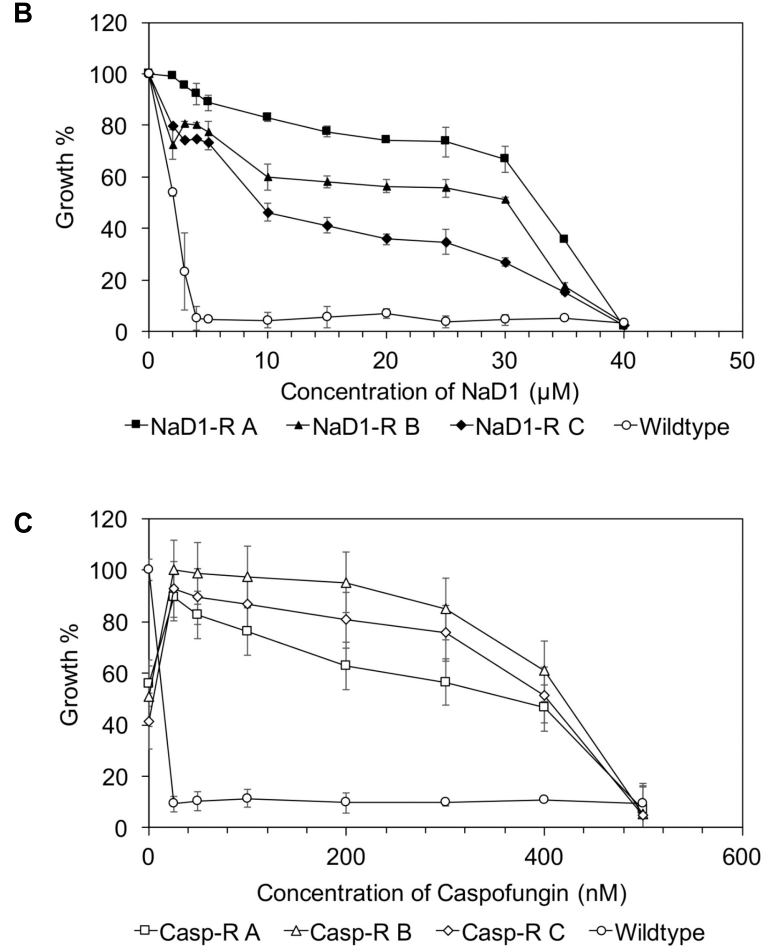

FIGURE 1 | Mild resistance to NaD1 or caspofungin can be evolved in vitro. Summary of the development of resistance during sub-culturing in the presence of Caspofungin or NaD1 (A). Three independent strains of NaD1-resistant yeast are shown, along with a representative example of the caspofungin-resistant lines. The antifungal activity of NaD1 (B) and caspofungin (C) against NaD1-resistant and caspofungin-resistant lines, respectively, is graphed relative to the highest measured OD for each strain. Error bars represent \pm standard error of the mean $(n=3)$.

was broad-spectrum or specific to NaD1. The caspofunginresistant strains were as sensitive to $\mathrm{NaD} 1$ as the wild type (Figure 2A), and similarly, the NaD1-resistant strains were as sensitive to caspofungin as the wild-type strain (Figure 2B).

$\mathrm{NaD} 1$-resistant strains were tested against some other plant defensins; NaD2 from Nicotiana alata, DmAmp1 from Dahlia mercki and the chimeric defensin HXP4. The NaD1-resistant strains were not resistant to NaD2 with an MIC of $20 \mu \mathrm{M}$, which was the same as the wild-type (Figure 3A). However, they were more resistant to DmAMP1 with an MIC of $20 \mu \mathrm{M}$ 

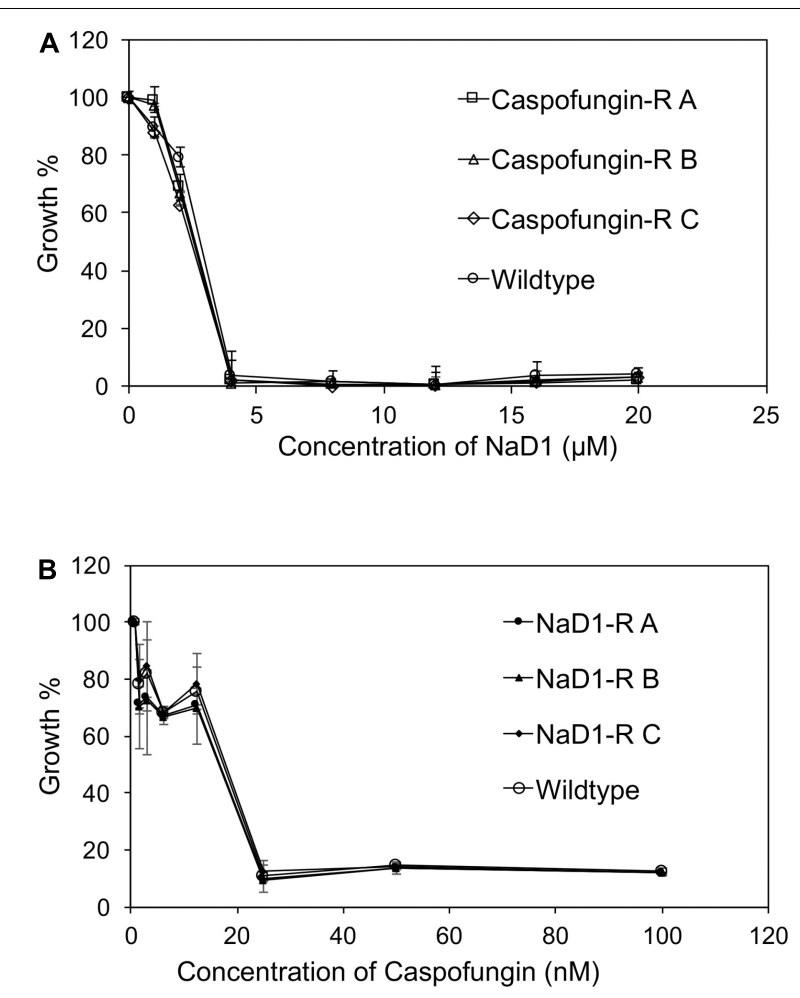

FIGURE 2 | There is no cross activity between caspofungin-resistance and NaD1-resistance. Cross-resistance of caspofungin-resistant (A) and NaD1-resistant lines (B), on NaD1 and caspofungin was determined, respectively. (A) Average growth percentage is shown, relative to the highest measured absorbance for each strain. Error bars represent \pm standard error of the mean $(n=3)$.

compared to $1.25 \mu \mathrm{M}$ for the wild type (Figure 3B) and to HXP4 with an MIC of $20 \mu \mathrm{M}$ compared to $10 \mu \mathrm{M}$ for the wild type (Figure 3C). Similarly, NaD1-resistant strains were not resistant to two unrelated cationic antifungal proteins, bovine pancreatic trypsin inhibitor (BPTI) and the insect cecropin CP29. The MIC for BPTI against both the wild type and the NaD1-resistant cultures was $10 \mu \mathrm{M}$ (Figure 3D). When incubated with CP29 the NaD1-resistant strains grew slightly better than wild-type at concentrations below the MIC, but the MIC was the same for all strains tested (Figure 3E).

\section{There Is a Fitness Penalty Associated With NaD1 Resistance}

The relative fitness of the $\mathrm{NaD} 1$-resistant strains was assessed by comparing growth rate over $48 \mathrm{~h}$ in two different growth media. NaD1-resistant strains B and C grew slower in YPD for the first $18 \mathrm{~h}$ but reached the same culture density as wild-type after $23 \mathrm{~h}$. NaD1-resistant strain A grew marginally slower than the wild type (Figure 4A). The growth in $1 / 2$ PDB was less varied, with only NaD1-resistant strain $\mathrm{C}$ growing significantly more slowly than wild-type (Figure 4B). The cellular dimensions of NaD1resistant strains were smaller than the wild type in both length and area (Figure 5).

\section{NaD1-Resistant Strains Are Sensitive to Cell Wall Stressors and Are Resistant to Osmotic Stress}

Potential alterations to the cell wall and membrane were examined by exposing the $\mathrm{NaD} 1$ resistant strains to SDS and CFW. SDS is an anionic detergent that causes cell wall stress, and membrane permeabilization and CFW is a cell wall stressor that binds to chitin. This revealed a significant growth defect of the NaD1-resistant strains in the presence of SDS or CFW (Figures 6B,C). Sensitivity of the NaD1-resistant strains was observed at $12.5 \mu \mathrm{g} / \mathrm{mL}$ SDS (Supplementary Figure 3) and at 1 $\mu \mathrm{g} / \mathrm{mL}$ CFW (Supplementary Figure 4).

In Candida albicans, the HOG1 osmotic stress response pathway is involved in tolerance to NaD1 (Hayes et al., 2013). It was, therefore, important to assess whether the $S$. cerevisiae $\mathrm{NaD} 1$-resistant strains had an altered osmotic stress response. $\mathrm{NaD} 1$-resistant strains grew better than wild-type at $200 \mathrm{mM}$ $\mathrm{NaCl}$ (Figure 6D and Supplementary Figure 5). This supported the hypothesis that NaD1-resistance correlates with increased osmotic stress tolerance.

\section{NaD1-Resistant Strains Are Not Resistant to Hydrogen Peroxide, UV Light, or Heat}

$\mathrm{NaD} 1$ induces ROS production in Candida albicans, which is a contributing factor to cell death. However, at low NaD1 levels, C. albicans cells cope by activation of the HOG1 pathway and enhancing transcription of genes that protect against oxidative stress (Hayes et al., 2013). Thus, the NaD1-resistant strains were tested for sensitivity to hydrogen peroxide generated oxidative stress. The NaD1-resistant strains grew the same as the wildtype strain in the presence of a range of hydrogen peroxide concentrations (Figure 7 and Supplementary Figure 6).

NaD1-resistant strains were also tested for resilience to ultraviolet light (UV) that causes DNA damage, as well as their resilience to heat shock.

There was no observable difference in the growth of the NaD1resistant strains and the wild-type cells after UV light or heat treatment (Supplementary Figure 7).

\section{Genetic Characterization of NaD1 Resistance}

The genomes of each of the NaD1-resistant and non-selected control lines were sequenced to identify mutations exclusively found in $\mathrm{NaD} 1$ resistant lines. Mutated genes identified in the resistant isolates were compared to the genes in the nonselected wild type (Table 2 ), along with the predicted amino acid changes. There were eight mutated genes found in NaD1resistant strain $\mathrm{A}$, five mutated genes in strain $\mathrm{B}$, and seven genes mutated in strain $\mathrm{C}$. There were three genes mutated in all three strains (FPS1, TOM1, and RSP5) and two genes were mutated in both NaD1-resistant B and C strains (PHO84 and CWP2) (Table 2). The results obtained from the VEP (McKenna et al., 2010), which determines the consequence of DNA variants on 

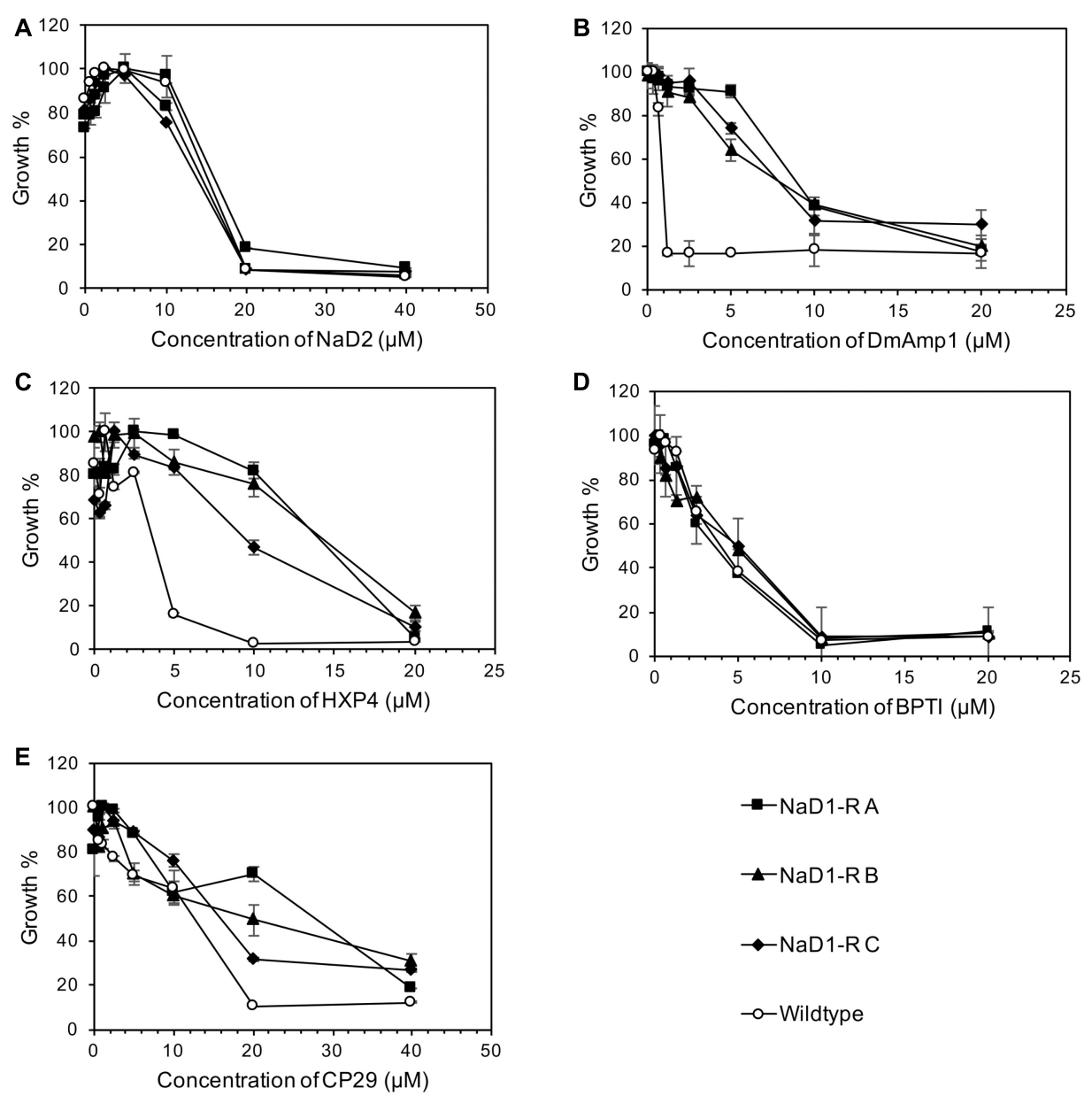

FIGURE 3 | Resistance to NaD1 is not broad spectrum. Growth inhibition of NaD1-resistant strains by a selection of antimicrobial peptides of different origin and mechanisms of action. The peptides examined were: the plant defensins NaD2 (A), DmAMP1 (B), and HXP4 (C), BPTI, a trypsin inhibitor from Bos Taurus (D), and the insect-derived cercropin variant CP29 (E). NaD1-resistant strains were also resistant to the plant defensins HXP4 and DmAMP1 but not to NaD2 or the antifungal BPTI. The three NaD1-resistant strains were slightly more resistant to CP29. Average growth percentage is shown, relative to the highest measured absorbance for each strain. Error bars represent \pm standard error of the mean $(n=3)$.

TABLE 2 | Summary of variants that disrupted protein coding regions in NaD1-R strains.

\begin{tabular}{llcll}
\hline Gene Name & Amino acid change & NaD1-R strains containing variant & Type & Inference \\
\hline BUD4 & p.Asn415Asp & A & SNV & Tolerated missense variant \\
CWP2 & p.Leu92del & B, C & INDEL & Disruptive in-frame deletion \\
FPS1 & p.Phe555fs & A, C & INDEL & Disruptive frame shift \\
MRPS16 & p.Pro45Gln & SNV & Deleterious missense variant \\
PHO84 & p.Ser183Phep.Val202lle & BC & SNV & Deleterious missense variant \\
PMR1 & p.Val170lle & A & SNV & Deleterious missense variant \\
RAS2 & p.Asp112Gly & A & SNV & Deleterious missense variant \\
RET2 & p.Gln12His & A, B, C & SNV & Missense variant \\
RSP5 & p.Gly689Cys & A & SNV & Missense variant \\
SIR3 & p.Glu451*STOP & A & SNV & Disruptive premature stop \\
SKY1 & p.Trp173Leu & A, B, C & SNV & Deleterious missense variant \\
TOM1 & p.Ala2381Gly & SNV & Deleterious missense variant
\end{tabular}

The observed changes to protein coding regions of NaD1- $R$ strains are shown along with the inferred impact on the encoded protein. SNV, single nucleotide variant; INDEL, insertion or deletion. These genes may be viewed on the Saccharomyces Genome Database www.yeastgenome.org (Cherry et al., 2012; Engel et al., 2014). 


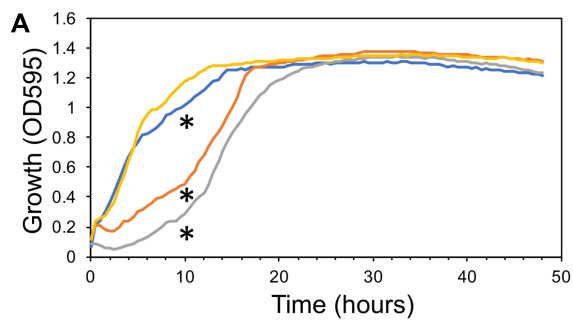

B

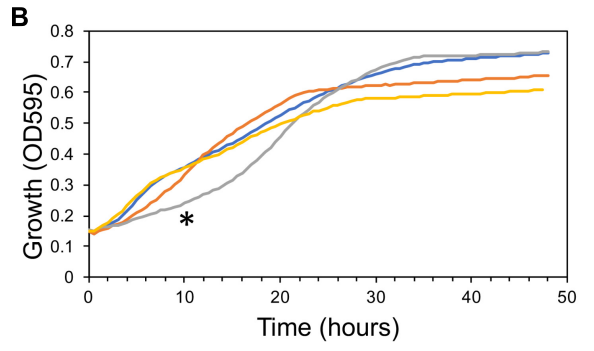

-NaD1-RA - NaD1-RB -NaD1-R C - Wildtype

FIGURE 4 | Growth rate of NaD1-resistant strains in YPD and 1/2 PDB medium compared to wild-type S. cerevisiae BY4741. Growth rate in the absence of antifungals was determined for NaD1-resistant strains in YPD medium (A) and $1 / 2$ PDB medium (B). An asterisk (*) denotes a statistically significant difference in growth rate compared to wild-type, by a two-tailed homoscedastic $T$-test $(P<0.05, n=6)$.

protein sequence, are listed in Table 2. A description of predicted functions for the affected genes is listed in Table 3.

\section{Determining the Relative Contributions of Loss of Function Mutations to NaD1 Tolerance}

It was considered likely that most of the observed mutations would have resulted in a loss-of-function phenotype for the

TABLE 3 | Summary of gene functions impacted by NaD1-resistance.

\begin{tabular}{lcr}
\hline Gene & Functional group & Description \\
\hline BUD4 & Cell wall & Protein involved in bud-site selection. \\
CWP2 & Cell wall & Cell wall mannoprotein. \\
FPS1 & Transport & Aquaglyceroporin, plasma membrane channel. \\
PHO84 & Transport & Inorganic phosphate transporter. \\
PMR1 & Transport & Calcium and manganese transport to the Golgi. \\
SKY1 & Signaling & Regulating cation homeostasis. \\
RAS2 & Signaling & Regulates sporulation and filamentous growth. \\
TOM1 & Ubiquitin ligase & E3 ubiquitin ligase (Hect-domain class) \\
RSP5 & Ubiquitin ligase & E3 ubiquitin ligase (NEDD4 family) \\
SIR3 & Chromatin binding & Chromatin remodeling. \\
RET2 & Unknown & Retrograde transport between Golgi and ER.
\end{tabular}

MRPS16 Ribosome structure

The genes that have mutations linked to NaD1 resistance and the description of their role in S. cerevisiae are shown. Gene ontology functional analysis revealed that some of these genes can be grouped by location or function, including: cell wall, transporter, signaling or ubiquitin ligase categories. These genes may be viewed on the Saccharomyces Genome Database www.yeastgenome.org (Cherry et al., 2012; Engel et al., 2014).
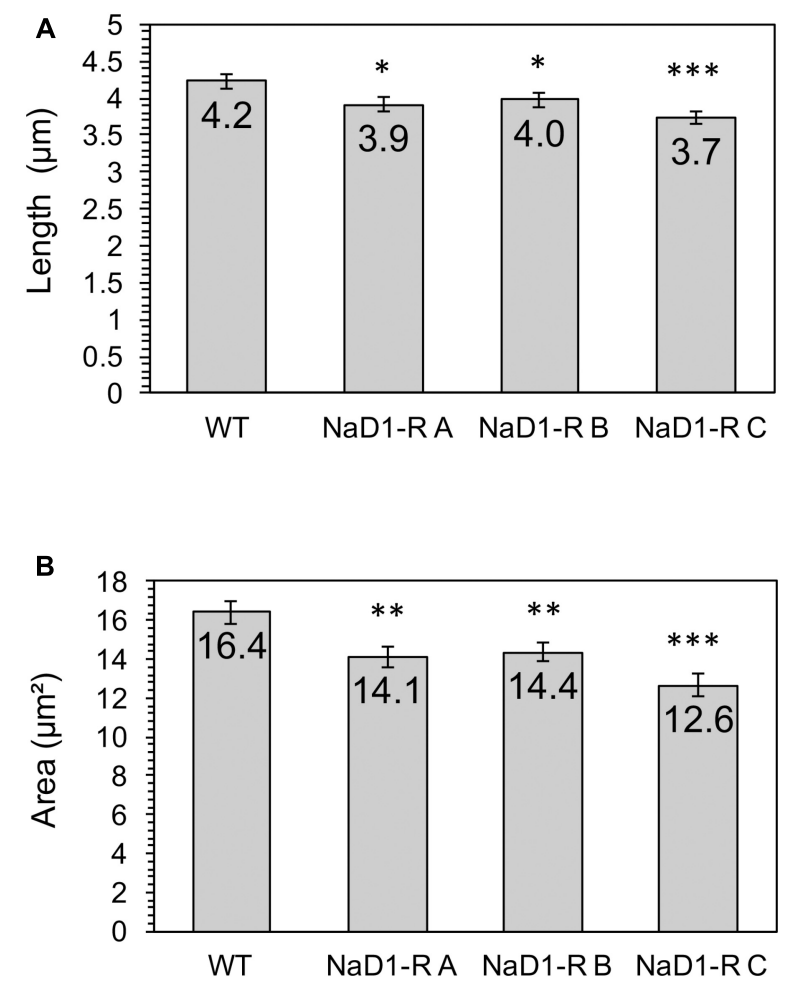

FIGURE 5 | NaD1-resistant strains are smaller in length and area compared to wild type. Cross-sectional length $\mathbf{( A )}$ and area (B) was determined for NaD1-resistant strains and wild-type. Average values are shown \pm 1 standard error of the mean $(n=30)$. $P$ value determined by two-tailed homoscedastic $T$-test indicated by $* P<0.05,{ }^{* *} P<0.01,{ }^{* * *} P<0.001$.

affected genes. To test this hypothesis, strains with single-gene knockouts for mutated genes were retrieved from the yeast deletion set (Winzeler et al., 1999) and antifungal growth assays were performed to assess whether gene deletion replicated the NaD1-resistant phenotype. The knock-out strains were only selected from non-essential genes. The antifungal assay revealed that none of the single gene knockout mutants ( $f p s 1 \Delta, c w p 2 \Delta$, $m r p s 16 \Delta$, pmr1 $\Delta$, pho84 $\Delta$, and sky1 $\Delta$ ) were as resistant to NaD1 as the three $\mathrm{NaD} 1$ resistant strains. Instead, each of the knockout mutations conferred partial resistance to NaD1. The highest level of resistance from a single knock-out occurred with $f p s 1 \Delta$, which had an MIC of $9 \mu \mathrm{M}$. Compared to the original NaD1-R mutants that had MICs of $40 \mu \mathrm{M}, c w p 2 \Delta$, pmr1 $\Delta, \operatorname{mrps} 16 \Delta$, and pho84 $\Delta$ contributed a smaller amount of resistance with an MIC of 6-7.5 $\mu \mathrm{M}$ while sky1 $\Delta$ had the same MIC as the wild-type and control strains (Table 4).

\section{DISCUSSION}

\section{Resistance to NaD1 Is Slow to Develop}

Antimicrobial peptides represent a promising next generation of therapeutics to combat drug-resistant fungi and bacteria (Wang et al., 2016). Peptides provide benefits as pharmaceuticals over small molecule drugs because they bind with high specificity to 


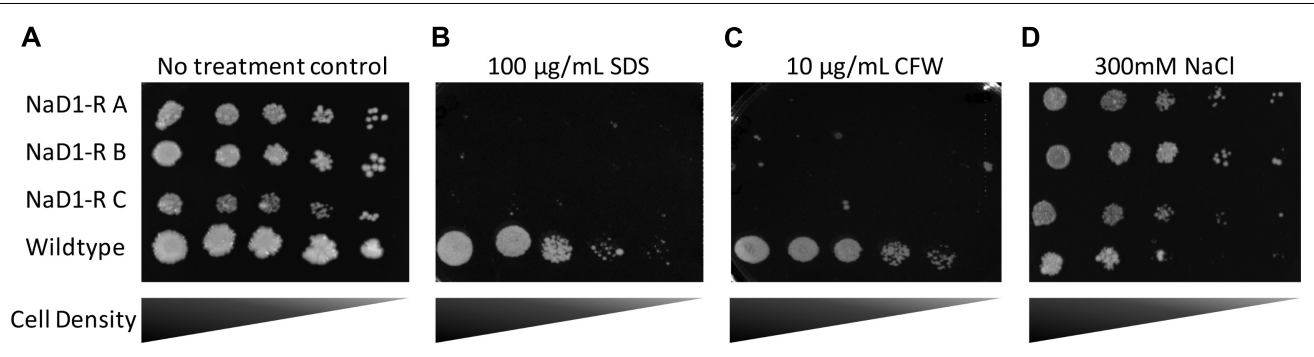

FIGURE 6 | NaD1-resistant strains are sensitive to SDS and calcofluor white (CFW), but are resistant to NaCl. NaD1-resistant and wild-type S. cerevisiae BY4741 cells were diluted and spotted onto YPD agar alone (A) or YPD supplemented with SDS (B), CFW (C), or NaCl (D). NaD1-resistant strains but not the wild type were inhibited by $100 \mu \mathrm{g} / \mathrm{mL}$ SDS or $10 \mu \mathrm{g} / \mathrm{mL}$ CFW compared to wild type. However, NaD1-resistant strains maintained growth at concentrations of NaCl that the wild type strains could not tolerate. Images are representative of three repeated experiments, all showing similar results.
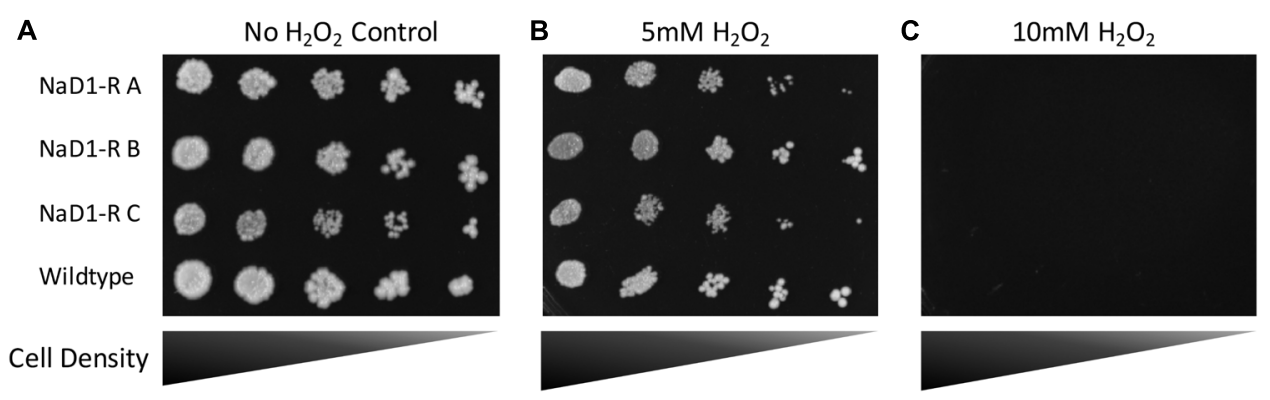

FIGURE 7 | NaD1-resistance does not alter sensitivity to oxidative stress. NaD1-resistant and wild-type S. cerevisiae BY4741 cells were diluted and spotted onto YPD agar containing $0 \mathrm{mM}(\mathbf{A}), 5 \mathrm{mM}(\mathbf{B})$, or $10 \mathrm{mM}(\mathbf{C}) \mathrm{H}_{2} \mathrm{O}_{2}$. There was no difference in $\mathrm{H}_{2} \mathrm{O}_{2}$ sensitivity between the NaD1-resistant strains compared to the wild type. Images are representative of three experiments, all showing similar results.

TABLE 4 | Comparison of NaD1 activity against single-gene deletion strains representing key resistance variants.

\begin{tabular}{lll}
\hline Strain of S. cerevisiae & NaD1 MIC $(\boldsymbol{\mu} \mathbf{M})$ & $\mathbf{9 5 \%} \mathbf{C l} \pm$ \\
\hline Wild type & 4.5 & 0.03 \\
NaD1-resistant strain A & 40 & 0.03 \\
NaD1-resistant strain B & 40 & 0.04 \\
NaD1-resistant strain C & 40 & 0.03 \\
Control A & 4.5 & 0.03 \\
Control B & 4.5 & 0.03 \\
Control C & 4.5 & 0.03 \\
FPS1 knockout & 9 & 0.05 \\
CWP2 knockout & 6 & 0.03 \\
MRPS16 knockout & 7.5 & 0.06 \\
PMR1 knockout & 6 & 0.03 \\
PHO84 knockout & 6 & 0.04 \\
SKY1 knockout & 4.5 & 0.02 \\
\hline
\end{tabular}

The minimum inhibitory concentration (MIC) of NaD1 was determined for single gene deletion strains linked to NaD1 resistance is shown, alongside control lines and the NaD1-resistant strains. Average values and a 95\% confidence interval (Cl) were calculated from three independent experiments.

their targets and require a relatively large interaction interface, which results in fewer off-target side effects (Craik et al., 2013). Plant defensins are known to bind to lipids and polysaccharides (Kvansakul et al., 2016; Payne et al., 2016; Poon et al., 2014). As hypothesized in this report, resistance to the antifungal peptide $\mathrm{NaD} 1$ developed more slowly than resistance to the small molecule drug caspofungin (Figure 1). The MIC of NaD1resistant strains was only 10 -fold greater than wild type, which was less than the equivalent caspofungin-resistant strains (20fold greater than wild type) and caspofungin resistance developed more rapidly than $\mathrm{NaD} 1$ resistance (Figure 1). Our observation is consistent with the reported benefits of peptide drugs, where their larger interaction surface requires more changes to the target before binding is disrupted.

\section{Resistance to NaD1 Did Not Confer Broad-Spectrum Resistance to Other Antifungal Peptides}

An example of broad-spectrum resistance to cationic AFPs has been reported for an agp $2 \Delta$ mutant of $S$. cerevisiae whereby resistance was mediated by an accumulation of positive charges at the cell surface that repelled positively charged antifungal peptides (Bleackley et al., 2014b). Therefore, it was important to determine whether evolved NaD1-resistant strains were resistant to other cationic peptides. The NaD1-resistant strains were resistant to the plant defensins HXP4 and DmAMP1 (Figures 3B,C). HXP4 is a chimera of $\mathrm{NaD} 1$ and $\mathrm{NaD} 2$, with a similar mechanism of action of $\mathrm{NaD} 1$, and hence was expected to share cross-resistance with NaD1 (Bleackley et al., 2016). DmAmp1 a plant defensin from Dahlia merckii, has a different mechanism of action to NaD1 (Parisi et al., 2018) whereby it 
binds to sphingolipids in the cell wall and plasma membrane of S. cerevisiae to exert antifungal activity (Thevissen et al., 2000). Although DmAMP1 and NaD1 have different mechanisms of action, they each stimulate the high-osmolarity glycerol (HOG) pathway in C. albicans (Hayes et al., 2013) and mutants in that pathway (hog1 or pbs2) were more sensitive to NaD1 and DmAmp1. In $S$. cerevisiae, the alteration of the osmotic stress pathway could also affect the sensitivity to DmAmp1. The antifungals BPTI and $\mathrm{NaD} 2$ were still effective against the NaD1resistant strains demonstrating the developed resistance was not broad spectrum (Figures 3A,D). BPTI inhibits $S$. cerevisiae growth by targeting a magnesium transporter and blocking the uptake of magnesium. Therefore, it was expected that the NaD1resistant strains would still be sensitive to BPTI (Bleackley et al., 2014a). The mechanism of action of NaD2 is mostly unknown, but it is known to bind to phosphatidic acid to exert its antifungal activity, unlike NaD1 that binds to both PIP2 and PA (Bleackley et al., 2016; Payne et al., 2016). The cationic peptide CP29 was less effective at sub MIC concentrations, but there was no shift in MIC detected (Figure 3E). Taken together this means that the resistance to NaD1 did not occur through a broad-spectrum resistance mechanism against all cationic AFPs. Plant defensins act synergistically with the clinical antifungal caspofungin and boost overall antifungal activity (van der Weerden et al., 2014; Vriens et al., 2015, 2016). We found that NaD1 was still effective against strains resistant to caspofungin (Figure 2A). Plant defensins may provide a very robust therapy if delivered in combination with existing clinical antifungals.

\section{Resistance to NaD1 Has a Fitness Penalty}

NaD1-resistant strains were tested for physical differences with wild type cells, to establish whether there is a fitness penalty associated with NaD1-resistance. The cell growth assays (Figure 4) revealed that NaD1-resistant strains grew more slowly than the wild type strain in the rich medium, YPD. NaD1resistant strain $\mathrm{C}$ grew the slowest in YPD medium, this may be due to the mutation in MRPS16, which is a mitochondrial ribosomal protein and RAS2, which regulates sporulation and filamentous growth. Knockout mutants of MRPS16 have been reported to have decreased vegetative and respiratory growth (Orij et al., 2012; Schlecht et al., 2014). A knockout of RAS2 has also been reported to have decreased fitness in YPD medium (Qian et al., 2012), supporting our observation that the NaD1resistant strain $\mathrm{C}$ had the largest fitness defect. Interestingly, the growth of the NaD1-resistant strains was equivalent to wild type in $1 / 2 \mathrm{PDB}$, supporting the veracity of the antifungal assays that were all performed in this medium. Individual cells of the NaD1-resistant strains were smaller in length and cross-sectional area compared to wild-type cells grown in YPD (Figure 5). $\mathrm{NaD} 1$-resistant strains were consequently tested against a range of cell wall stressors to investigate whether adaptations to $\mathrm{NaD} 1$ resistance had altered the properties of the cell wall and membrane. SDS is a detergent with a negatively charged head group that is commonly used to test the susceptibility of yeast cells to membrane permeabilization and cell wall perturbation
(Sirisattha et al., 2004; Gao et al., 2014). NaD1-resistant strains were more sensitive to SDS than the wild type strain (Figure 6). This sensitivity suggests that strains with enhanced tolerance to $\mathrm{NaD} 1$ have modifications their cell walls or plasma membranes, and may be more susceptible to alternative antifungal drugs. NaD1-resistant strains were also sensitive to CFW, which binds to cell wall chitin and leads to permeabilization (Figure 6). NaD1-resistant strains may contain more chitin in their cell wall and therefore increase the binding of CFW (Roncero et al., 1988). Furthermore, CFW relies on a functional Hog1 pathway for its antifungal activity, and thus hyperactive Hog1 signaling to protect against defensin activity may result in heightened sensitivity to CFW (García-Rodriguez et al., 2000). NaD1-resistant strains were also tested against more diverse environmental stresses, but we found no evidence for protection or sensitivity to oxidative stress (Figure 7), DNA damage or heat stress. We have established a link between $\mathrm{NaD} 1$ resistance and cell wall stress. The observed $\mathrm{NaD} 1$ resistance appears limited to the NaD1 mechanism of action, and it does not mitigate oxidative stress, DNA damage or heat shock.

\section{Unlike Resistance to Azoles and Echinocandins, Resistance to NaD1 Occurs via Multiple Quantitative Mutations}

Whole genome sequencing revealed multiple genes were linked to $\mathrm{NaD} 1$ resistance (Table 2 ). The functional diversity of these genes revealed that the mechanism of $\mathrm{NaD} 1$ is likely to involve more than a single protein target. This contrasts with resistance to caspofungin, which can be achieved by a single amino acid alteration in the targeted $\beta$-glucan synthase Fkslp (Katiyar and Edlind, 2009), or resistance to fluconazole with mutations to the Erg11p enzyme (Sionov et al., 2012). Our caspofungin-resistant mutants all followed this path to resistance, with each acquiring a single mutation at residue 639 of Fks1p. The NaD1-resistant strains acquired mutations related to the protection from osmotic stress, alteration of the cell wall, solute transport, signaling, and cation homeostasis (Table 3). In summary, unlike echinocandin and azole classes of fungicides, resistance to $\mathrm{NaD} 1$ did not feature a "hot-spot" for genomic mutations.

The NaD1-resistant strains had accumulated several mutations, and thus no single gene could be identified that was responsible for the resistance phenotype. The relative contribution of each observed mutation was assessed by comparing the level of $\mathrm{NaD} 1$ resistance in strains with knockouts of individual genes (Table 4). None of the single gene knockouts produced the level of $\mathrm{NaD} 1$ resistance obtained in the evolved strains. The FPS1 knockout had the biggest effect and was mutated in all three of the evolved resistant strains. PHO84, PMR1, and CWP2 deletion mutants contributed relatively smaller degrees of $\mathrm{NaD} 1$ resistance. In PHO84 and PMR1, mutations in the NaD1-resistant strains were single nucleotide changes with conservative effects; it may be that protein function was only mildly affected. Combinations of mutations were not assessed as we felt that an exhaustive account of these variants was not supported 
Glycerol
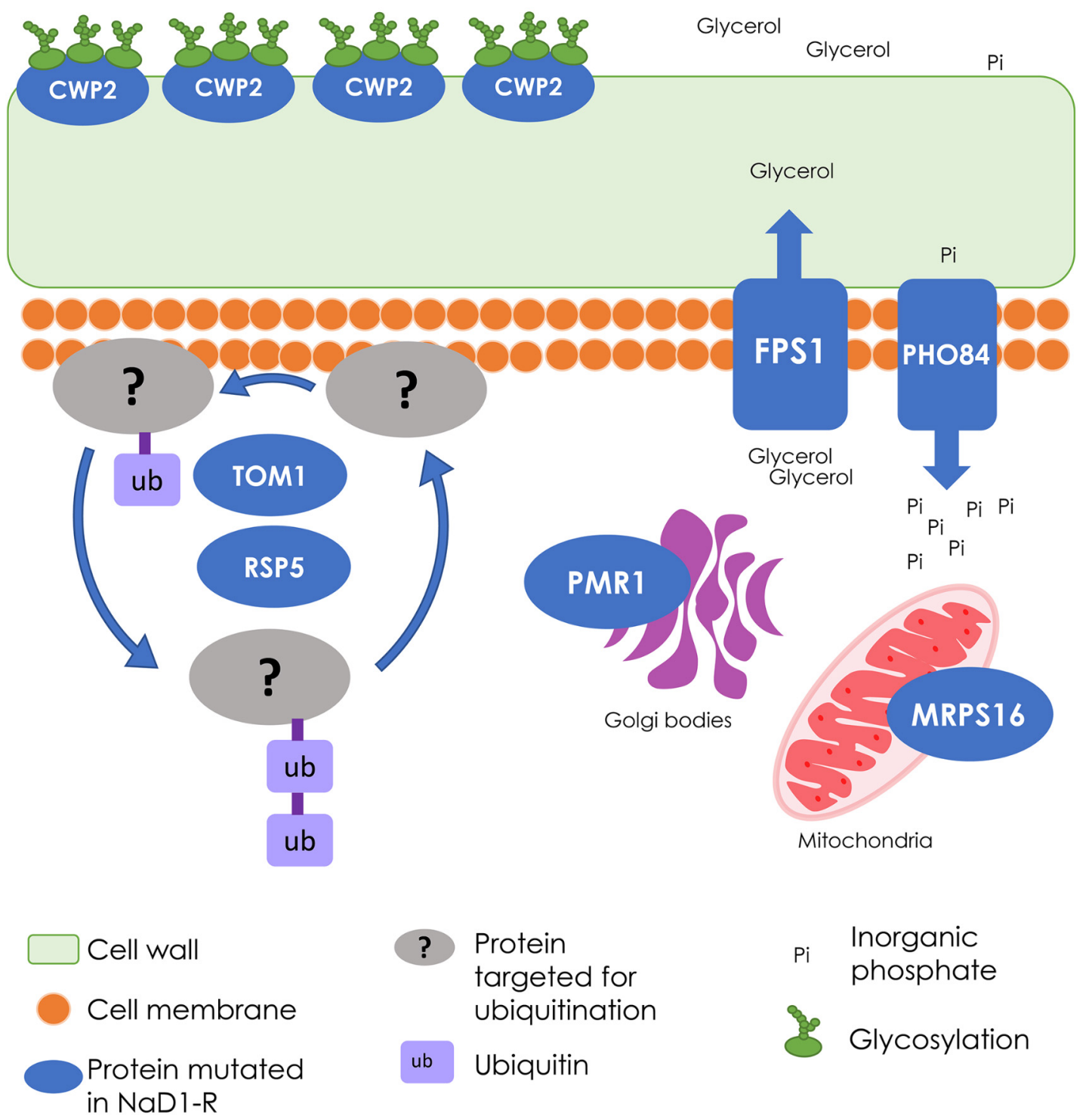

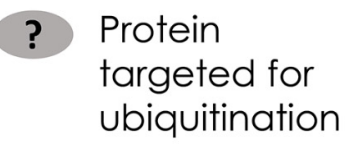

ub Ubiquitin

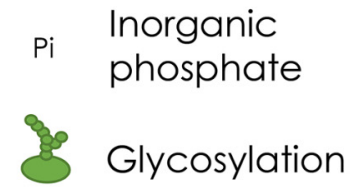

FIGURE 8 | Summary of the wild-type cellular processes that were modified in NaD1-resistant strains. The cellular processes involved in NaD1 resistance included the cell wall, transport, and signaling functions. FPS1 is a glycerol transporter that protects the cell from osmotic shock by exporting or preventing export of glycerol; the FPS1 gene deletion was the most resistant single-gene knockout tested. Other gene deletions that contributed to NaD1 resistance were: CWP2, which is a cell wall mannoprotein, PHO84, which is a phosphate and manganese ion transporter, PMR1, which transports calcium and manganese to the Golgi bodies, and MRPS16, which is a mitochondrial ribosomal protein. TOM1 and RSP5 are essential ubiquitin ligases that were mutated in all NaD1-resistant strains. Ubiquitin ligases target proteins for degradation and regulate a range of processes, including multivesicular body sorting. Overall, these genes regulate a range of cellular processes with a common theme of cell wall and osmo-regulation.

as we did not have enough individual strains to support which combination was evolutionarily more successful. Future work will focus on increasing the number of individual resistant lines studied. This should provide quantitative data on the relative benefit of different combinations of variants.

There were SNV's found in the TOM1 and RSP5 genes of all three NaD1-resistant strains. The SNV's are unlikely to lead to a complete loss of function and instead are likely to represent a partial loss or gain of function. Both TOM1 and RSP5 are E3 ubiquitin ligases, a class of protein that tags protein substrates for destruction. Ubiquitin ligases regulate diverse functions including cell trafficking, DNA repair, and signaling. TOM1 regulates mRNA export from the nucleus and targets excess histones for degradation (Saleh et al., 1998; Singh et al., 2009). RSP5 is an essential gene that regulates a variety of processes including mitochondrion organization and sorting of multivesicular bodies (Katzmann et al., 2004; McNatt 
et al., 2007; Kaliszewski and Zoladek, 2008). In C. albicans, $\mathrm{NaD} 1$ is known to cross the plasma membrane via endocytosis. It is possible that a restriction of multivesicular transport could also restrict $\mathrm{NaD} 1$ movement inside the target cell. It has also been reported that decreased function of RSP5 can increase the susceptibility to cell wall stressors such as calcofluor, as was seen for $\mathrm{NaD} 1$-resistant lines in our stress assays (Figure 6). The identification of genomic variants in essential genes highlights an advantage of natural selection and genome sequencing as a method to identify mechanisms of resistance mechanisms.

\section{Resistance to NaD1 Has a Common Theme of the Osmotic Stress Response}

In our study, FPS1 was mutated in all three of the evolved NaD1resistant strains. FPS1 encodes an aquaglyceroporin plasma membrane channel with a role in the efflux of glycerol and xylitol (Luyten et al., 1995). This efflux pump maintains osmotic balance by moderating the passive diffusion of glycerol (Toh et al., 2001). NaD1-resistant strains all contained a frameshift (Phe555fs) that prevents translation of 115 amino acids from the C-terminal regulatory domain of the FPS1 protein (Hedfalk et al., 2004). This could result in substantial modification to its function and cellular osmotic balance because this 115amino acid region contains seven phosphorylation sites and two ubiquitinylated lysine sites that regulate the function of the channel. It is unclear if loss of this c-terminal region would cause protein instability and a total loss of function, or if it would produce an unregulated glycerol channel. The phenotype of the FPS1 knockout had significant resistance to $\mathrm{NaD} 1$, suggesting loss of function is the most likely result of the frameshift mutation. Fpslp is regulated by the HOG pathway in S. cerevisiae. In wild-type cells the Fps1p-mediated efflux of glycerol decreases when the cell is under hyper-osmotic (high salt) stress which in turn increases the internal accumulation of glycerol (Hedfalk et al., 2004). In theory, the FPS1 deletion mutants will be resistant to hyper-osmotic shock as they are always accumulating intracellular glycerol (Toh et al., 2001). This resistance to hyper-osmotic stress was confirmed in the $\mathrm{NaCl}$ spot assays where we observed increased growth of NaD1-resistant strains under high salt conditions compared to wild-type cells (Figure 6D). We hypothesize that loss of FPS1 activity would prevent the release of excess turgor pressure via glycerol efflux, and result in excess pressure on the cell wall and susceptibility to cell wall stress. This is supported by previous reports that show that a combination of a FPS1 deletion with cell wall weakening mutations in S. cerevisiae results in cell lysis and lethality (Tamás et al., 1999). In work by García-Rodriguez et al. (2000), the ability of CFW to inhibit S. cerevisiae was dependent on a functional HOG pathway (Figure 7C). The work of Hayes et al. (2013) in Candida albicans supports this model as $\mathrm{NaD} 1$ is known to activate the osmotic stress response, or HOG, pathway in C. albicans and permit tolerance of low amounts of $\mathrm{NaD} 1$. In addition, hog1 mutants are more sensitive to $\mathrm{NaD} 1$ and DmAmp1 (Hayes et al., 2013). In a similar mechanism, via modification of the osmotic balance of the cell, our yeast mutants gained resistance to plant defensins NaD1 and DmAMP1 and conversely increased their sensitivity to cell wall stressors. The role of FPS1 in resistance to NaD1 is consistent with $\mathrm{NaD} 1$ activation of Hog1p in C. albicans, as FPS1p activity is regulated by Hoglp in S. cerevisiae (Lee et al., 2013; Muir et al., 2015). One possible mechanism for FPS1-mediated NaD1 resistance is that FPS1 mutants accumulate high intracellular concentrations of glycerol, which stabilizes lipid bilayers and protects the cellular organelles that are targeted by the NaD1 protein.

The NaD1-resistant strains also had mutations in other solute transporters. $\mathrm{PHO} 84$ an inorganic phosphate transporter and low affinity manganese transporter and, PMR1 a high affinity calcium and manganese transporter (Lapinskas et al., 1995; Jensen et al., 2003). Calcium is known to be involved in the response to osmotic stress, S. cerevisiae releases a stretch-activated pulse of calcium ions in response to cellular swelling from hypo-osmotic stress (Batiza et al., 1996; Tong et al., 2004). It is possible the Pmrlp transporter produces this calcium release.

NaD1-resistant strains also had mutations in genes that affect cell wall composition including CWP2, RAS2, and BUD4. CWP2 encodes a mannoprotein that has a major role in stabilizing the cell wall (Frieman and Cormack, 2003). Mutants with a cwp2 deletion are more sensitive to CFW and congo red, which are cell wall stressors, providing another explanation for why the NaD1-resistant strains were more sensitive to $\mathrm{CFW}$ than the wild type in Figure 7C (van der Vaart et al., 1995). Both RAS2 and BUD4 affect the structure of the cell wall and are associated with protein localization to the bud neck (Gimeno et al., 1992; Kang et al., 2013). Hence, changes in cell size and growth in the resistant strains (Figures 5, 6) could be linked to the mutations in these genes. In summary, the NaD1-resistant mutants were characterized by mutations that increased resistance to hyperosmotic stress and conversely increased sensitivity of the resistant strains to cell wall stressors such as CFW and SDS. An overall summary of the key changes observed in NaD1-resistant strains is presented in Figure 8.

\section{CONCLUSION}

In this paper, we described the development of $S$. cerevisiae tolerance to an antifungal plant protein, the defensin NaD1. The overall aim was to compare the rate and mechanism of resistance development of a small protein to a small molecule antifungal of the echinocandin class. This study identified that resistance to the defensin $\mathrm{NaD} 1$ was slow to develop and had limited effectiveness compared to caspofungin resistance. A fitness penalty was associated with $\mathrm{NaD} 1$ resistance, thus if the selective pressure of $\mathrm{NaD} 1$ was removed it is likely that nonresistant strains would outcompete the $\mathrm{NaD} 1$ resistant strains. Increased tolerance to $\mathrm{NaD} 1$ developed via the accumulation of multiple mutations over time, and not via a single target site modification as with caspofungin. There was no cross resistance observed between $\mathrm{NaD} 1$ or caspofungin resistance, 
therefore, this study indicates that $\mathrm{NaD} 1$, and by extension other plant defensins, may complement existing clinical antifungals due to their resilience and unique mechanism of action.

\section{AUTHOR CONTRIBUTIONS}

AM performed the experiments and wrote the manuscript. $\mathrm{MB}, \mathrm{MA}$, and $\mathrm{RL}$ edited the manuscript and designed the experiments.

\section{REFERENCES}

Batiza, A. F., Schulz, T., and Masson, P. H. (1996). Yeast respond to hypotonic shock with a calcium pulse. J. Biol. Chem. 271, 23357-23362. doi: 10.1074/jbc. 271.38.23357

Berkut, A. A., Usmanova, D. R., Peigneur, S., Oparin, P. B., Mineev, K. S., Odintsova, T. I., et al. (2014). Structural similarity between defense peptide from wheat and scorpion neurotoxin permits rational functional design. J. Biol. Chem. 289, 14331-14340. doi: 10.1074/jbc.M113.530477

Bleackley, M. R., Hayes, B. M., Parisi, K., Saiyed, T., Traven, A., Potter, I. D., et al. (2014a). Bovine pancreatic trypsin inhibitor is a new antifungal peptide that inhibits cellular magnesium uptake. Mol. Microbiol. 92, 1188-1197. doi: $10.1111 / \mathrm{mmi} .12621$

Bleackley, M. R., Payne, J. A. E., Hayes, B. M. E., Durek, T., Craik, D. J., Shafee, T. M. A., et al. (2016). Nicotiana alata defensin chimeras reveal differences in the mechanism of fungal and tumor cell killing and an enhanced antifungal variant. Antimicrob. Agents Chemother. 60, 6302-6312. doi: 10.1128/aac. 01479-16

Bleackley, M. R., Wiltshire, J. L., Perrine-Walker, F., Vasa, S., Burns, R. L., van der Weerden, N. L., et al. (2014b). Agp2p, the plasma membrane transregulator of polyamine uptake, regulates the antifungal activities of the plant defensin NaD1 and other cationic peptides. Antimicrob. Agents Chemother. 58, 2688-2698. doi: 10.1128/aac.02087-13

Cherry, J. M., Hong, E. L., Amundsen, C., Balakrishnan, R., Binkley, G., Chan, E. T., et al. (2012). Saccharomyces genome database: the genomics resource of budding yeast. Nucleic Acids Res. 40, D700-D705. doi: 10.1093/nar/ gkr1029

Cingolani, P., Platts, A., Wang le, L., Coon, M., Nguyen, T., Wang, L., et al. (2012). A program for annotating and predicting the effects of single nucleotide polymorphisms, SnpEff: SNPs in the genome of Drosophila melanogaster strain w1118; iso-2; iso-3. Fly 6, 80-92. doi: 10.4161/fly.19695

Craik, D. J., Fairlie, D. P., Liras, S., and Price, D. (2013). The future of peptide-based drugs. Chem. Biol. Drug Des. 81, 136-147. doi: 10.1111/cbdd. 12055

Dracatos, P. M., van der Weerden, N. L., Carroll, K. T., Johnson, E. D., Plummer, K. M., and Anderson, M. A. (2014). Inhibition of cereal rust fungi by both class I and II defensins derived from the flowers of Nicotiana alata. Mol. Plant Pathol. 15, 67-79. doi: 10.1111/mpp.12066

Engel, S. R., Dietrich, F. S., Fisk, D. G., Binkley, G., Balakrishnan, R., Costanzo, M. C., et al. (2014). The reference genome sequence of Saccharomyces cerevisiae: then and now. G3 4, 389-398. doi: 10.1534/g3.113. 008995

Frieman, M. B., and Cormack, B. P. (2003). The omega-site sequence of glycosylphosphatidylinositol-anchored proteins in Saccharomyces cerevisiae can determine distribution between the membrane and the cell wall. Mol. Microbiol. 50, 883-896. doi: 10.1046/j.1365-2958.2003. 03722.x

Gao, Q., Liou, L. C., Ren, Q., Bao, X., and Zhang, Z. (2014). Salt stress causes cell wall damage in yeast cells lacking mitochondrial DNA. Microb. Cell 1, 94-99. doi: 10.15698/mic2014.01.131

García-Rodriguez, L. J., Durán, A., and Roncero, C. (2000). Calcofluor antifungal action depends on chitin and a functional high-osmolarity glycerol response (HOG) pathway: evidence for a physiological role of the Saccharomyces

\section{FUNDING}

This study was funded by an Australian Research Council grant DP 160100309.

\section{SUPPLEMENTARY MATERIAL}

The Supplementary Material for this article can be found online at: https://www.frontiersin.org/articles/10.3389/fmicb. 2018.01648/full\#supplementary-material

cerevisiae HOG pathway under non-inducing conditions. J. Bacteriol. 182, 2428-2437. doi: 10.1128/JB.182.9.2428-2437.2000

Gimeno, C. J., Ljungdahl, P. O., Styles, C. A., and Fink, G. R. (1992). Unipolar cell divisions in the yeast $S$. cerevisiae lead to filamentous growth: regulation by starvation and RAS. Cell 68, 1077-1090. doi: 10.1016/0092-8674(92) 90079-R

Hayes, B. M., Anderson, M. A., Traven, A., van der Weerden, N. L., and Bleackley, M. R. (2014). Activation of stress signalling pathways enhances tolerance of fungi to chemical fungicides and antifungal proteins. Cell. Mol. Life Sci. 71, 2651-2666. doi: 10.1007/s00018-014-1573-8

Hayes, B. M. E., Bleackley, M. R., Wiltshire, J. L., Anderson, M. A., Traven, A., and van der Weerden, N. L. (2013). Identification and mechanism of action of the plant defensin $\mathrm{NaD} 1$ as a new member of the antifungal drug arsenal against Candida albicans. Antimicrob. Agents Chemother. 57, 3667-3675. doi: 10.1128/aac.00365-13

Hedfalk, K., Bill, R. M., Mullins, J. G., Karlgren, S., Filipsson, C., Bergstrom, J., et al. (2004). A regulatory domain in the C-terminal extension of the yeast glycerol channel Fps1p. J. Biol. Chem. 279, 14954-14960. doi: 10.1074/jbc.M31312 6200

Jensen, L. T., Ajua-Alemanji, M., and Culotta, V. C. (2003). The Saccharomyces cerevisiae high affinity phosphate transporter encoded by $\mathrm{PHO} 84$ also functions in manganese homeostasis. J. Biol. Chem. 278, 42036-42040. doi: 10.1074/jbc. M307413200

Kaliszewski, P., and Zoladek, T. (2008). The role of Rsp5 ubiquitin ligase in regulation of diverse processes in yeast cells. Acta Biochim. Pol. 55, 649-662.

Kang, P. J., Hood-DeGrenier, J. K., and Park, H. O. (2013). Coupling of septins to the axial landmark by BUD4 in budding yeast. J. Cell Sci. 126, 1218-1226. doi: $10.1242 /$ jcs. 118521

Katiyar, S. K., and Edlind, T. D. (2009). Role for Fks1 in the intrinsic echinocandin resistance of Fusarium solani as evidenced by hybrid expression in Saccharomyces cerevisiae. Antimicrob. Agents Chemother. 53, 1772-1778. doi: 10.1128/AAC.00020-09

Katzmann, D. J., Sarkar, S., Chu, T., Audhya, A., and Emr, S. D. (2004). Multivesicular body sorting: ubiquitin ligase Rsp5 is required for the modification and sorting of carboxypeptidase S. Mol. Biol. Cell 15, 468-480. doi: 10.1091/mbc.E03-07-0473

Kvansakul, M., Lay, F. T., Adda, C. G., Veneer, P. K., Baxter, A. A., Phan, T. K., et al. (2016). Binding of phosphatidic acid by NsD7 mediates the formation of helical defensin-lipid oligomeric assemblies and membrane permeabilization. Proc. Natl. Acad. Sci. U.S.A. 113, 11202-11207. doi: 10.1073/pnas.160785 5113

Lapinskas, P. J., Cunningham, K. W., Liu, X. F., Fink, G. R., and Culotta, V. C. (1995). Mutations in PMR1 suppress oxidative damage in yeast cells lacking superoxide dismutase. Mol. Cell. Biol. 15, 1382-1388. doi: 10.1128/MCB.15.3. 1382

Lass-Flörl, C. (2009). The changing face of epidemiology of invasive fungal disease in Europe. Mycoses 52, 197-205. doi: 10.1111/j.1439-0507.2009. 01691.x

Lay, F. T., Brugliera, F., and Anderson, M. A. (2003). Isolation and properties of floral defensins from ornamental tobacco and petunia. Plant Phys. 131, 1283-1293. doi: 10.1104/pp.102.016626

Lay, F. T., Mills, G. D., Hulett, M. D., and Kvansakul, M. (2012). Crystallization and preliminary X-ray crystallographic analysis of the plant defensin NaD1. 
Acta Crystallogr. F Struct. Biol. Cryst. Commun. 68, 85-88. doi: 10.1107/ S1744309111049530

Lee, J., Reiter, W., Dohnal, I., Gregori, C., Beese-Sims, S., Kuchler, K., et al. (2013). MAPK Hogl closes the $S$. cerevisiae glycerol channel Fps1 by phosphorylating and displacing its positive regulators. Gene Dev. 27, 2590-2601. doi: 10.1101/ gad.229310.113

Leroux, P., Fritz, R., Debieu, D., Albertini, C., Lanen, C., Bach, J., et al. (2002). Mechanisms of resistance to fungicides in field strains of Botrytis cinerea. Pest Manag. Sci. 58, 876-888. doi: 10.1002/ps.566

Luyten, K., Albertyn, J., Skibbe, W. F., Prior, B. A., Ramos, J., Thevelein, J. M., et al. (1995). Fps1, a yeast member of the MIP family of channel proteins, is a facilitator for glycerol uptake and efflux and is inactive under osmotic stress. EMBO J. 14, 1360-1371.

McKenna, A., Hanna, M., Banks, E., Sivachenko, A., Cibulskis, K., Kernytsky, A., et al. (2010). The genome analysis toolkit: a MapReduce framework for analyzing next-generation DNA sequencing data. Genome Res. 20, 1297-1303. doi: $10.1101 /$ gr.107524.110

McLaren, W., Gil, L., Hunt, S. E., Riat, H. S., Ritchie, G. R. S., Thormann, A. et al. (2016). The ensembl variant effect predictor. Genome Biol. 17:122. doi: 10.1186/s13059-016-0974-4

McNatt, M. W., McKittrick, I., West, M., and Odorizzi, G. (2007). ). Direct binding to Rsp5 mediates ubiquitin-independent sorting of Sna3 via the multivesicular body pathway. Mol. Biol. Cell 18, 697-706. doi: 10.1091/mbc.e06-080663

Muir, A., Roelants, F. M., Timmons, G., Leskoske, K. L., and Thorner, J. (2015). Down-regulation of TORC2-Ypk1 signaling promotes MAPK-independent survival under hyperosmotic stress. eLife 4:e09336. doi: 10.7554/eLife. 09336

Murray, G. M., and Brennan, J. P. (2009). Estimating disease losses to the Australian wheat industry. Aust. Plant. Pathol. 38, 558-570. doi: 10.1071/ ap09053

Orij, R., Urbanus, M. L., Vizeacoumar, F. J., Giaever, G., Boone, C., Nislow, C., et al. (2012). Genome-wide analysis of intracellular $\mathrm{pH}$ reveals quantitative control of cell division rate by $\mathrm{pH}$ in Saccharomyces cerevisiae. Genome Biol. 13:R80. doi: 10.1186/gb-2012-13-9-r80

Ortega, M., Marco, F., Soriano, A., Almela, M., Martínez, J. A., Pitart, C., et al. (2010). Candida spp. bloodstream infection: influence of antifungal treatment on outcome. J. Antimicrob. Chemother. 65, 562-568. doi: 10.1093/jac/ dkp495

Parisi, K., Shafee, T. M. A., Quimbar, P., van der Weerden, N. L., Bleackley, M. R., and Anderson, M. A. (2018). The evolution, function and mechanisms of action for plant defensins. Semin. Cell Dev. Biol. doi: 10.1016/j.semcdb.2018.02.004 [Epub ahead of print].

Payne, J. A., Bleackley, M. R., Lee, T. H., Shafee, T. M., Poon, I. K., Hulett, M. D., et al. (2016). The plant defensin NaD1 introduces membrane disorder through a specific interaction with the lipid, phosphatidylinositol 4,5 bisphosphate. Biochim. Biophys. Acta 1858, 1099-1109. doi: 10.1016/j.bbamem.2016. 02.016

Poon, I. K. H., Baxter, A. A., Lay, F. T., Mills, G. D., Adda, C. G., Payne, J. A. E., et al. (2014). Phosphoinositide-mediated oligomerization of a defensin induces cell lysis. eLife 3:e01808. doi: 10.7554/eLife.01808

Qian, W., Ma, D., Xiao, C., Wang, Z., and Zhang, J. (2012). The genomic landscape and evolutionary resolution of antagonistic pleiotropy in yeast. Cell Rep. 2, 1399-1410. doi: 10.1016/j.celrep.2012.09.017

Robinson, J. T., Thorvaldsdóttir, H., Winckler, W., Guttman, M., Lander, E. S., Getz, G., et al. (2011). Integrative genomics viewer. Nat. Biotechnol. 29, 24-26. doi: $10.1038 /$ nbt. 1754

Roncero, C., Valdivieso, M. H., Ribas, J. C., and Durán, A. (1988). Isolation and characterization of Saccharomyces cerevisiae mutants resistant to Calcofluor white. J. Bacteriol. 170, 1950-1954. doi: 10.1128/jb.170.4.1950-1954. 1988

Sagaram, U. S., Pandurangi, R., Kaur, J., Smith, T. J., and Shah, D. M. (2011). Structure-activity determinants in antifungal plant defensins MsDEF1 and MtDEF4 with different modes of action against Fusarium graminearum. PLoS One 6:e18550. doi: 10.1371/journal.pone. 0018550

Sanglard, D., Ischer, F., Monod, M., and Bille, J. (1996). Susceptibilities of Candida albicans multidrug transporter mutants to various antifungal agents and other metabolic inhibitors. Antimicrob. Agents Chemother. 40, 2300-2305.

Saleh, A., Collart, M., Martens, J. A., Genereaux, J., Allard, S., Cote', J., et al. (1998). TOM1p, a yeast hect-domain protein which mediates transcriptional regulation through the ADA/SAGA coactivator complexes. J. Mol. Biol. 282, 933-946. doi: 10.1006/jmbi.1998.2036

Schindelin, J., Arganda-Carreras, I., Frise, E., Kaynig, V., Longair, M., Pietzsch, T., et al. (2012). Fiji: an open-source platform for biological-image analysis. Nat. Methods 9, 676-682. doi: 10.1038/nmeth.2019

Schlecht, U., Suresh, S., Xu, W., Aparicio, A. M., Chu, A., Proctor, M. J., et al. (2014). A functional screen for copper homeostasis genes identifies a pharmacologically tractable cellular system. BMC Genomics 15:263. doi: 10 . 1186/1471-2164-15-263

Sherry, S. T., Ward, M.-H., Kholodov, M., Baker, J., Phan, L., Smigielski, E. M., et al. (2001). dbSNP: the NCBI database of genetic variation. Nucleic Acid Res. 29, 308-311. doi: 10.1093/nar/29.1.308

Singh, R. K., Kabbaj, M. H., Paik, J., Gunjan, A. (2009). Histone levels are regulated by phosphorylation and ubiquitylation-dependent proteolysis. Nat. Cell Biol. 11, 925-933. doi: 10.1038/ncb1903

Sionov, E., Chang, Y. C., Garraffo, H. M., Dolan, M. A., Ghannoum, M. A., and Kwon-Chung, K. J. (2012). Identification of a Cryptococcus neoformans Cytochrome P450 lanosterol 14 $\alpha$-demethylase (ERG11) residue critical for differential susceptibility between fluconazole/voriconazole and itraconazole/posaconazole. Antimicrob. Agents Chemother. 56, 1162-1169. doi: 10.1128/aac.05502-11

Sirisattha, S., Momose, Y., Kitagawa, E., and Iwahashi, H. (2004). Toxicity of anionic detergents determined by Saccharomyces cerevisiae microarray analysis. Water Res. 38, 61-70. doi: 10.1016/j.watres.2003. 08.027

Tamás, M. J., Luyten, K., Sutherland, F. C. W., Hernandez, A., Albertyn, J., Valadi, H., et al. (1999). Fpslp controls the accumulation and release of the compatible solute glycerol in yeast osmoregulation. Mol. Microbiol. 31, 10871104. doi: 10.1046/j.1365-2958.1999.01248.x

Thevissen, K., Osborn, R. W., Acland, D. P., and Broekaert, W. F. (2000). Specific binding sites for an antifungal plant defensin from Dahlia (Dahlia merckii) on fungal cells are required for antifungal activity. Mol. Plant Microbe Interact. 13, 54-61. doi: 10.1094/MPMI.2000.13.1.54

Toh, T. H., Kayingo, G., van der Merwe, M. J., Kilian, S. G., Hallsworth, J. E., Hohmann, S., et al. (2001). Implications of FPS1 deletion and membrane ergosterol content for glycerol efflux from Saccharomyces cerevisiae. FEMS Yeast Res. 1, 205-211.

Tong, A. H., Lesage, G., Bader, G. D., Ding, H., Xu, H., Xin, X., et al. (2004). Global mapping of the yeast genetic interaction network. Science 303, 808-813. doi: 10.1126/science.1091317

van der Vaart, J. M., Caro, L. H., Chapman, J. W., Klis, F. M., and Verrips, C. T. (1995). Identification of three mannoproteins in the cell wall of Saccharomyces cerevisiae. J. Bacteriol. 177, 3104-3110. doi: 10.1128/jb.177.11.3104-3110. 1995

van der Weerden, N. L., Anderson, M. A., and Payne, J. A. (2014). Agents and Methods of Treatment. U.S. Patent No 20170042966A1. Washington, DC: U.S Patent and Trademark Office.

van der Weerden, N. L., Bleackley, M. R., and Anderson, M. A. (2013). Properties and mechanisms of action of naturally occurring antifungal peptides. Cell. Mol. Life Sci. 70, 3545-3570. doi: 10.1007/s00018-0131260-1

van der Weerden, N. L., Hancock, R. E. W., and Anderson, M. A. (2010). Permeabilization of fungal hyphae by the plant defensin NaD1 occurs through a cell wall-dependent process. J. Biol. Chem. 285, 37513-37520. doi: 10.1074/jbc. M110.134882

van der Weerden, N. L., Lay, F. T., and Anderson, M. A. (2008). The plant defensin, $\mathrm{NaD} 1$, enters the cytoplasm of Fusarium oxysporum hyphae. J. Biol. Chem. 283, 14445-14452. doi: 10.1074/jbc.M709867200

Verweij, P. E., Snelders, E., Kema, G. H., Mellado, E., and Melchers, W. J. (2009). Azole resistance in Aspergillus fumigatus: a side-effect of environmental fungicide use? Lancet Infect. Dis. 9, 789-795. doi: 10.1016/s1473-3099(09) 70265-8

Vriens, K., Cools, T. L., Harvey, P. J., Craik, D. J., Braem, A., Vleugels, J., et al. (2016). The radish defensins RsAFP1 and RsAFP2 act synergistically with 
caspofungin against Candida albicans biofilms. Peptides 75, 71-79. doi: 10.1016/ j.peptides.2015.11.001

Vriens, K., Cools, T. L., Harvey, P. J., Craik, D. J., Spincemaille, P., Cassiman, D., et al. (2015). Synergistic activity of the plant defensin HsAFP1 and caspofungin against Candida albicans biofilms and planktonic cultures. PLoS One 10:e132701. doi: 10.1371/journal.pone.0132701

Wang, C. K., King, G. J., Conibear, A. C., Ramos, M. C., Chaousis, S., Henriques, S. T., et al. (2016). Mirror images of antimicrobial peptides provide reflections on their functions and amyloidogenic properties. J. Am. Chem. Soc. 138, 5706-5713. doi: 10.1021/jacs.6b02575

Winzeler, E. A., Shoemaker, D. D., Astromoff, A., Liang, H., Anderson, K., Andre, B., et al. (1999). Functional characterization of the S. cerevisiae genome by gene deletion and parallel analysis. Science 285, 901-906. doi: 10.1126/ science.285.5429.901
Conflict of Interest Statement: MA is the Executive Director and Chief Scientific Officer of Hexima Ltd.

The remaining authors declare that the research was conducted in the absence of any commercial or financial relationships that could be construed as a potential conflict of interest.

Copyright (C) 2018 McColl, Bleackley, Anderson and Lowe. This is an open-access article distributed under the terms of the Creative Commons Attribution License (CC BY). The use, distribution or reproduction in other forums is permitted, provided the original author(s) and the copyright owner(s) are credited and that the original publication in this journal is cited, in accordance with accepted academic practice. No use, distribution or reproduction is permitted which does not comply with these terms. 